\title{
Movilidad en el medio construido. Una mirada desde la cognición espacial ${ }^{1}$
}

\author{
Rodrigo Mora \\ Universidad Técnica Federico Santa María, Valparaíso, Chile. \\ Email: rodrigo.mora@usm.cl \\ Pablo Isla \\ Universidad Técnica Federico Santa María, Valparaíso, Chile.
}

\section{Agustín Ibáñez}

Universidad Diego Portales, Santiago, Chile.

\begin{abstract}
Resumen: Este artículo revisa aportes recientes en el campo de las ciencias cognitivas en relación al diseño del hábitat construido. Primeramente se describen los principales paradigmas teóricos que han animado el debate sobre cognición y conducta humanas durante las últimas décadas, para luego examinar las posibles aplicaciones de estas teorías en la arquitectura y el urbanismo, particularmente en torno a la capacidad de orientarnos y movernos en el medio construido.
\end{abstract}

Palabras clave: dinámica de la cognición, arquitectura, comportamiento

\section{Mobility in the built environment. A view from spatial cognition}

\begin{abstract}
This article reviews recent contributions in the field of cognitive science in relation to the design of the built environment. First we describe the main theoretical paradigms that have animated the debate on human cognition and behavior over the past decades, and then discuss possible applications of these theories in architecture and urbanism, particularly around our ability to orient and move in the built environment.
\end{abstract}

Key words: dynamics of cognition, architecture, behavior

\section{Mobilidade no ambiente construído. Uma visão desde a cognição espacial}

Resumo: Este artigo analisa as contribuições recentes no campo da ciência cognitiva em relação ao desenho do ambiente construído. Primeiro vamos descrever os principais paradigmas teóricos que têm animado o debate sobre cognição e comportamento humano nas últimas décadas e, em seguida, discutir as possíveis aplicações dessas teorias em arquitetura e urbanismo, especialmente em torno da 
capacidade de orientar e se mover em ambiente construído.

Palavras-chave: dinâmica da cognição, arquitetura, comportamento

$* * *$

\section{Introducción}

Las ciencias dedicadas al estudio de la cognición humana comparten con las disciplinas dedicadas al diseño del hábitat, como la arquitectura y planificación urbana principalmente, la premisa fundamental de que el comportamiento humano está influenciado por el medio físico. Mientras para los arquitectos y planificadores urbanos el comportamiento humano es influenciado (vagamente quizás), por la forma en que los volúmenes se articulan entre sí, en el caso de la arquitectura, o la forma de ordenamiento del sistema de calles o espacios públicos, en el caso del urbanismo, para los estudiosos de la cognición, en especial los que vienen de la sicología, el comportamiento surge de procesos mentales de diversa naturaleza cuyo origen está en, o son afectados por, el medio físico. Por cierto, esta desconexión parece no ser exclusiva. Tal como plantean Hillier \& Vaughan (2007), arquitectos y planificadores urbanos por una parte, y sociólogos y cientistas sociales por otra, han históricamente visto la ciudad desde sus propios ámbitos disciplinares: mientras los primeros han en varios períodos visto la ciudad como agente del reforma social a través de creación de la utopía urbana (utopía presente en varios períodos históricos, desde la ciudad jardín de Ebenezer Howard de finales del siglo XX, hasta las propuestas futuristas y modernistas de Sant'Elia y Le Corbusier respectivamente, o las posturas contemporáneas del Nuevo Urbanismo de Andrés Duany), mientras los segundos han tendido a ver en el espacio construido el reflejo de una estructura económica desigual a través de la cual se perpetúa el sistema capitalista de acumulación (Soja 1989). Sin embargo, esta realidad esta comenzado a cambiar.

Las ciencias cognitivas están actualmente cada vez más interesadas en los entornos, sean estos sociales o materiales, donde se produce el comportamiento. Esto porque varias investigaciones han mostrado que en el medio físico se encuentran muchas de las claves de los fenómenos cognitivos que dan origen al comportamiento humano. Así, el cabal entendimiento del comportamiento, especialmente el que concierne a la navegación en el medio físico, debe necesariamente incorporar al medio construido como variable interviniente. La apuesta parece ser ver al medio construido no como el receptáculo donde la acción humana originada en la mente se lleva a cabo, sino como un agente que modifica esa acción en forma constante. Por su parte, la teoría urbana, especialmente la de origen anglosajón, ha empezado a interesarse cada vez más por los fenómenos cognitivos detrás de procesos tales como la orientación o la representación mental del medio construido. Si bien es cierto que ya en los sesenta se destacaba la necesidad de incorporar aspectos cognitivos en la planificación urbana 
(Lynch 1960), no es menos real que para la mayoría de los arquitectos estas ideas fueron internalizadas principalmente en el ámbito metodológico, con escasas ramificaciones a la teoría arquitectónica y urbana. No obstante, durante los últimos veinte años, y especialmente la última década, aportes provenientes de la teoría urbana propiamente tal (Alexander, 1988; Marshall, 2004; Salingaros, 2005; Batty 2005), y de las ciencias cognitivas (Portugali, 2004; Claramunt and Winter, 2007; Höelscher et al, 2007) parecen estar convergiendo en abordar el problema mente-espacio desde una mirada más holística.

Este es el tema de este artículo. Acá se discute cómo las teorías cognitivas contemporáneas permiten entender una de las conductas más ubicuas y necesarias de las personas: la capacidad de orientarse en el medio construido y de explorarlo (lo que en inglés se conoce como navigation). Para ello, la primera parte del artículo presentará una breve introducción sobre los orígenes de la cognición espacial. A continuación se hace una revisión de las principales corrientes cognitivas contemporáneas, las llamadas escuelas “dinámicas” (Ibañez, 2007b), para finalmente terminar subrayando cómo estas visiones se pueden incorporar al diseño del medio construido.

\section{La idea de mapa cognitivo}

La posibilidad de explicar cómo la gente se orienta y se mueve en el espacio ha sido un tema de discusión científica desde hace al menos cincuenta años. Al respecto, una disciplina conocida como cognición espacial, surgida del cruce entre geografía y sicología en los años cincuenta, pero cuyo desarrollo como tal comienza en los setenta, años ha intentado dar luces sobre en esta materia. Sus orígenes se remontan al trabajo de Edward Tolman, sicólogo americano que en 1948 publicó un artículo llamado "mapas cognitivos en ratas y hombres" y que describe una serie de experimentos en el que observó como las ratas se movían en escenarios artificiales.

En el más conocido de sus experimentos, varias ratas fueron dispuestas en un espacio circular desde el cual salía un pasillo largo que, después de una serie de cambios de dirección, terminaba en un lugar donde había comida. Los animales, que estaban hambrientos luego de varios días sin comer, comenzaban a explorar el sistema espacial, lo que era meticulosamente registrado por Tolman.

$\mathrm{Al}$ principio las ratas se demoraron en explorar este y otros pasillos. Con el tiempo, sin embargo, las ratas se aventuraron en el pasillo más largo, descubriendo al final de éste el alimento. Este aprendizaje hizo que al cabo de dos o tres experimentos, las ratas se dirigieran rápidamente al final del pasillo en cuanto eran colocadas en el mundo. Tolman entonces cambió el escenario, bloqueando el camino que antes conducía a la comida y poniendo en cambio muchos pasillos radiales (ver fase 2). Para su sorpresa, descubrió que la mayoría de las ratas intentaban alcanzar el espacio donde 
antes estaba la comida, prefiriendo un pasillo lateral (ver flecha) que aproximadamente terminaba en la ubicación de éste. Concluyó así que las ratas parecían haber formado un "mapa mental” o "mapa cognitivo" del espacio 2 , representación mental de tipo alocéntrica, es decir, como si el mundo fuera visto por un pájaro (Gärling, Böök et al., 1986; Golledge, 1992).

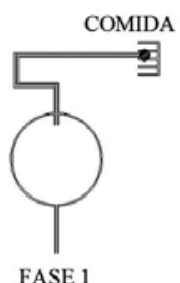

FASE 1

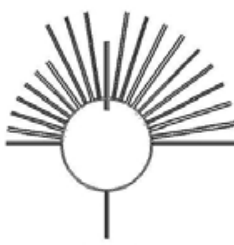

FASE 2

Figura 1: El más famoso experimento de Tolman. La imagen de la derecha muestra el camino preferido por las ratas en la fase 2 del experimento

Las ideas de Tolman fueron fundamentales para el desarrollo de la cognición espacial. Se comenzó así a hablar de "mapa cognitivo” para referirse a la representación mental del mundo de tipo abstracto (Lynch, 1960; Siegel \& White, 1973; Golledge et al 1985), cuya característica más fundamental es la de proveer información relacional del espacio. En la práctica, esto significa que un mapa cognitivo es capaz de dar información sobre la posición relativa de lugares y objetos en el mundo desde un marco de referencia externo al usuario. Así, la mayoría de los santiaguinos son capaces de saber la posición de, por ejemplo, el cerro San Cristóbal en relación a la cordillera de los Andes, independientemente de estar mirando hacia el norte o sur.

Dentro de la teoría urbanística propiamente tal, son pocos los referentes teóricos que aluden a la idea de mapa cognitivo. Quizás el precedente teórico más familiar para arquitectos y diseñadores sea el trabajo de Kevin Lynch (1960). Lynch sostenía que las ciudades poseen una "imagen”, y que ésta ere el resultado de la coordinación entre varios elementos; hitos, sendas, 'nodos, barrios y bordes. En la medida que exista armonía entre éstos, afirmaba Lynch, una imagen sería retenida mentalmente, lo que a su vez permitiría a las personas orientarse y moverse más eficientemente en las ciudades.

Otro referente más actual también relacionado con el ámbito arquitectónico es el trabajo de Kaplan (1982). Kaplan sugería que, para mantener el interés de las personas por explorar un medio dado, es necesario un balance entre la legibilidad del medio ambiente y su impredictabilidad. Conocida como la teoría del misterio, las ideas de Kaplan enfatizan el rol que tiene la curiosidad, expresada en el deseo de comprender algo (una 
edificio, por ejemplo) sobre lo cual tenemos posibles respuestas, en fomentar la exploración espacial.

Importantes para la disciplina arquitectónica son los trabajos de Alexander (1988) y Salingaros (2005). El primero postula que la ciudad no es un árbol, es decir, una estructura jerárquica organizada en torno a un tronco único común, sino un sistema de tramas de distinto nivel jerárquico que posibilitan ciertos grados de flexibilidad 'para la navegación humana. Esto porque para ir de un lugar a otro, las personas dispondrían, la mayoría de las veces de más de una alternativas, lo que a su vez permitiría que un mapa cognitivo emergiera en forma más natural en la mente de los habitantes. Por último, el trabajo de Salingaros (2005) es especialmente relevante para la teoría arquitectónica por cuanto toca especialmente la relación entre mente y medio construido. De acuerdo con Salingaros, la mente humana naturalmente busca información y significado en el medio construido. Los arquitectos y planificadores urbanos, sugiere, históricamente se han concentrado en lo segundo, descuidando el hecho que las personas, en forma natural e inconsciente, reciben y procesan información visual del medio, detonándose así una serie de comportamientos espaciales de distinto tipo. A veces, estos comportamientos nos permiten arribar exitosamente a nuestras destinaciones, pero otras, sugiere Salingaros, lo contrario sucede y nos perdemos en ciudades y edificios, a veces en forma reiterada.

Las ideas anteriores muestran hasta qué punto la idea de mapa cognitivo ha comenzado a permear la teoría arquitectónica y urbana en los últimos treinta años. Al respecto, dos son los aspectos fundamentales a resolver en lo relativo a los mapas cognitivos: cuáles son los procesos mentales que realizan las personas para representar el medio construido, y cómo funciona el proceso de toma de decisiones para que las personas se orienten y se muevan en este medio. Históricamente, la respuesta a estas dos interrogantes ha dependido cual es la construcción meta teórica dominante, es decir, la concepción sobre la naturaleza fundamental de la mente humana y sus procesos mayormente aceptada por los estudiosos del tema. A continuación se presenta algunas de los antecedentes teóricos y metodológicos que han fundamentado las respuestas a las dos principales interrogantes de la cognición espacial

\section{Una mirada histórica a las ciencias cognitivas}

El contexto actual de las ciencias cognitivas puede describirse como el de un cuestionamiento del paradigma tradicionalmente usado por los científicos para entender los procesos cognitivos: el computacionalismo. En su núcleo conceptual, este paradigma asumió que la mente podría ser entendida en base a cómputos simbólicos, siendo explicada en base a reglas y principios lógico-sintácticos. Conforme al avance del tiempo, la propia investigación dentro del computacionalismo fue provocando algunos insights acerca de las limitaciones del programa. Esto porque existe una multiplicidad de problemas meta-teóricos, metodológicos, conceptuales y 
empíricos a los que esta visión no podía responder.

En los últimos años el horizonte de las ciencias cognitivas parece estar teñido de crisis, o al menos de desencanto, con la empresa computacionalista (Cosmelli \& Ibáñez, 2008; Ibáñez \& Cosmelli, 2008). Esto ha hecho emerger enfoques que escapan a los supuestos computacionales y que han abierto el campo de investigación hacia fenómenos no abordados por la tradición ortodoxa, generando nuevos diálogos con otras disciplinas sociales y biológicas. Acá se mostraran algunos de esos enfoques.

La cognición situada (Brigthon et al. 2003; entre otros) asume que el conocimiento está estructurado en el ambiente social, ecológico y físico que conforma la experiencia. La embodied cognition, (Anderson 2003; entre otros), por otro lado, afirma que las propiedades de la mente (pensamientos, deseos, imaginación, etc.) están encarnadas en la misma corporalidad, que a su vez se encuentra insertada en un contexto ecológico particular (Cornejo et al. 2008; Ibáñez 2008; Ibáñez et al. 2006). Dentro de la embodied cognition se sitúa la lingüística cognitiva (Lakoff \& Johnson, 1999; entre otros) y la enacción (Thompson \& Varela 2001). La extended mind (Clark \& Chalmers, 1998; entre otros) está interesada en el rol del ambiente en la génesis de los procesos cognitivos, poniendo énfasis en la consideración de éste no sólo como parte de la acción, sino también del pensamiento. Así, mapas, herramientas y símbolos son parte de la mente extendida. La perspectiva de la cognición distribuida (Hutchins 1995; entre otros), sugiere que el conocimiento es tomado en un continuum con los procesos del ambiente y en ciertas ocasiones resulta complejo establecer distinciones tajantes entre mundo y mente. Finalmente la teoría de la actividad (Engeström 1991; entre otros) critica la aplicación de la simulación computacional para la comprensión de la cognición humana, suponiendo en cambio que fenómenos psicológicos como la intencionalidad no pueden ser entendidos en términos mecanicistas.

Las llamadas teorías dinámicas asumen muchos de los supuestos anteriores. El núcleo central de estas teorías está basado en la metáfora de la cognición como sistema dinámico (Ibáñez 2007a, 2007b, 2008; Ibáñez et al. 2009). Los enfoques dinámicos de la cognición acentúan la naturaleza ecológica y contextual de la cognición, enraizada en la interacción individuo-ambiente. A la vez, asumen que la mente no se basa en la manipulación de operaciones abstractas, sino en la convergencia de diversos procesos múltiples orientados por la acción del individuo en su contexto. Estos enfoques prometen (de forma controversial quizás) ser aplicados tanto a las interacciones neurales como a los fenómenos culturales, apoyando sus explicaciones en formalizaciones matemáticas de los fenómenos cognitivos, y promoviendo una imagen diferente de la metáfora de la computación clásica, más multifacética e inclusiva de los afectos y la cultura.

En lo relacionado a la movilidad en el medio construido, los modelos dinámicos contemporáneos muestran que claves internas y externas, 
múltiples y simultaneas, se entrelazan dinámicamente en la navegación espacial (Muir \& Taube 2002). Igualmente, investigaciones recientes en neurociencias sugieren que las neuronas "espaciales" no utilizan un mapa representacional del espacio abstracto y estático, sino más bien una mapa que depende de la acción situada específica y un contexto también específico (ver figura 2). Asimismo, estos estudios sugieren que múltiples procesos cognitivos y diferentes áreas cerebrales son coordinados cuando las personas razonan o actúan en ambientes espaciales (Burgess 2008).

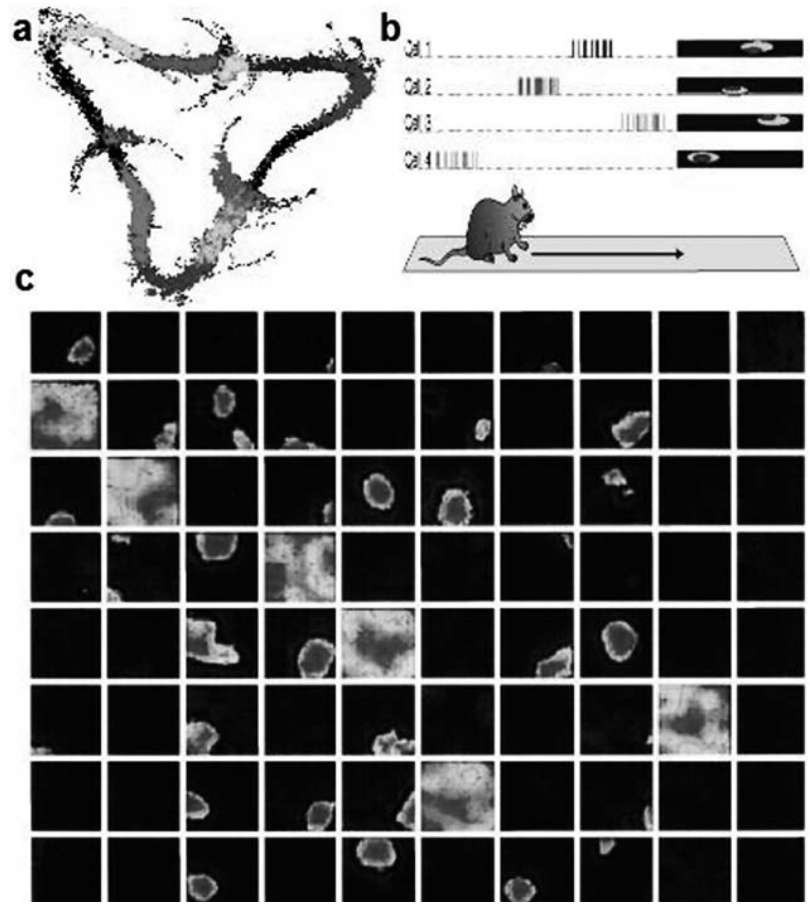

Figura 2: a) Representación topográfica de 7 neuronas del hipocampo (un área del cerebro asociada entre otras cosas a navegación especial y memoria) de una rata que se encuentra corriendo en un circuito triangular. Los puntos negros representan posiciones, el color es indicativo de la tasa de descarga neuronal, y cada neurona está representada por un color. b) representación de neuronas espaciales del hipocampo que reactivan cuando una rata se desplaza en línea recta. c) Mapas de 80 neuronas registradas simultáneamente en el hipocampo de la rata cuando se encuentra en movimiento. Dicha activación no se corresponde con un mapa estático del ambiente, pues la activación de estas neuronas depende de la actividad particular de la rata. A su vez, la activación relativa cambia en diferentes ambientes y actividades, mostrando una actividad no-topográfica de las neuronas espaciales. En síntesis, cada mapa depende de una nueva acción y situación (adaptado de Nakazawa et al., 2004). 


\section{Teorías cognitivas contemporáneas y el medio ambiente construido}

Para entender de mejor manera la idea de cognición situada, matriz común de las teorías contemporáneas de la cognición, se mostrará el siguiente ejemplo. La figura 3 muestra un experimento realizado recientemente, en el que se mostró a 42 estudiantes (26 hombres, 16 mujeres) universitarios un plano abstracto del campus principal de la Universidad Técnica Federico Santa María en Valparaíso. En el plano se mostraban cuatro puntos: X, Y, A y B. Los estudiantes debían en forma individual trazar con un lápiz cuál sería la ruta que harían para ir desde X a Y y luego de A a B. Una vez terminada esta tarea, los estudiantes fueron llevados uno por uno al lugar en cuestión y colocados en el punto A (que no tenía ninguna marca que pudiese ser reconocible), desde el cual debía dirigirse hacia el punto B (que tampoco tenía ningún tipo de señal), donde una tercera persona les saludaba con la mano. Una vez que llegaban a ese destino, debían retornar hasta el punto A, desde donde eran llevados hasta el punto X para repetir el procedimiento con el punto Y. Tanto los dibujos como las trayectorias de cada persona fueron registradas por el investigador a cargo.

Los resultados mostraron que, para el caso de la ruta A-B, solo el $21.4 \%$ de los encuestados realizó en terreno el camino trazado en el papel, mientras que el $52.4 \%$ hizo lo mismo en el caso de la ruta X-Y (Mora 2010). Se constató además que no existía coincidencia entre las rutas ocupadas para ir y para volver de un lugar, como lo demuestra el hecho que solo el 54.8\% de los encuestados realizaron lo mismo para el caso de la ruta A-B y el 69\% para el caso de la ruta X-Y.
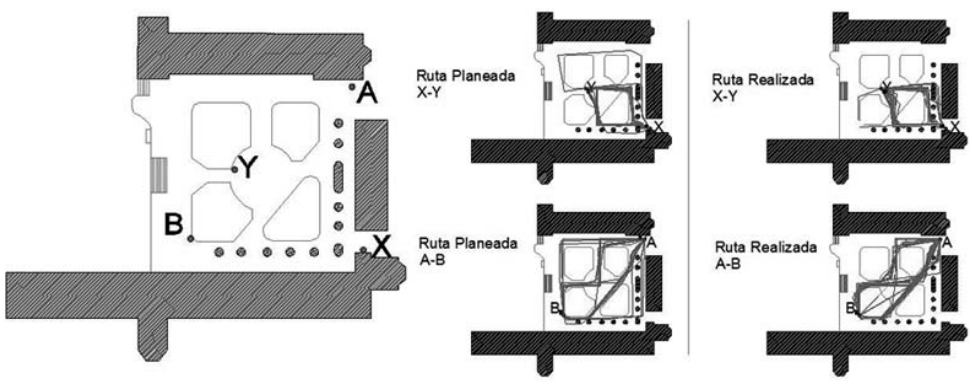

Figura 3: rutas trazadas y empleadas el interior del campus principal de la Universidad Técnica Federico Santa María. A diferencia de las dos figuras superiores, donde se detectó poca variación entre las rutas planeadas y realizadas, en las figuras inferiores las personas tendieron a realizar en la realidad rutas diferentes a las trazadas en el papel. Sin embargo, en ambos casos es posible ver que los caminos de ida son diferentes a los de vuelta.

Los resultados anteriores están en línea con experimentos realizados a nivel internacional (Golledge 1995, Conroy-Dalton 2003), que han 
detectado que el movimiento peatonal es afectado en forma importante por las condiciones existentes en el medio al momento de que éste es realizado. En este sentido, se podría hablar de una ecología del movimiento, cuya raíz está centrada en la percepción visual (Gibson, 1979) Ahora bien, el lector puede estar preguntándose ¿Cómo establecer un modelo teórico explicativo del comportamiento humano si aceptamos que éste es fundamentalmente situado, dependiente de las características del medio ambiente y que éste, a su vez, es hasta cierto punto impredecible?

Acá se propone que, el menos en lo concerniente a la arquitectura, una línea de pensamiento interesante de Hillier (1996), que sugiere que la navegación humana está íntimamente ligada a la visión. Heredero de los postulados de Gibson (1979) y Benedikt (1979), Hillier propone que para que las personas puedan navegar eficientemente y orientarse en el mundo debe existir una "redundancia de información visual”, es decir, la capacidad de ser capaz de ver mucho más allá que el espacio que se habita.

En el fondo, se trata de ser capaz de ver hacia adelante lo más posible, pues ese proceso implícitamente formaría en nosotros, en forma gradual, una "imagen mental" del medio construido. Esta imagen, a su vez, nos permitiría planear y realizar en forma eficiente rutas en ciudades y edificios, permitiéndonos idear mentalmente rutas nuevas cuando las usuales están inutilizables. En el fondo, la redundancia visual permitiría hacer frente a un entorno hasta cierto punto cambiante como es el medio físico.

Las ideas de Hillier pueden observadas en el siguiente experimento (figura 4). En él se pidió a un grupo de treinta personas navegar durante diez minutos dos mundos virtuales que se asemejaban a una ciudad (ConroyDalton, 2003). La única diferencia entre ambos mundos era el hecho que mientras en el primero existían continuidades visuales a lo largo de las calles (como en la mayor parte de las ciudades), en el segundo estas continuidades eran interrumpidas constantemente, de forma tal que no era posible formarse una idea clara de lo que sucedía "más allá” del espacio en que se estaba ocupando. Los resultados mostraron que en el primer mundo parecía surgir naturalmente la idea de “jerarquía espacial” en el sentido de que por algunas calles circulaba mucha gente, por otras una cantidad media de gente y por varias muy poca o ninguna persona. A diferencia de lo anterior, en el segundo mundo las trayectorias ocuparon prácticamente todo el espacio disponible, reflejando así una falta jerarquía espacial. Quizás más importante aún, mientras en el primer mundo las personas tendieron a detenerse en las esquinas (donde podían acceder a vasta información del entorno para seguir o enmendar su marcha), en el segundo, estas detenciones eran erráticas y muchas veces a mitad de cuadra, tal como hacemos cuando estamos desorientados y no sabemos dónde encontrar información que nos ayude (al respecto, véase Figura 4). Parecía así que las características visuales del medio eran claves a la hora de explicar el comportamiento de las personas, lo que vendría a explicar porqué la mayoría de las ciudades son sistemas relativamente estructurados y jerárquicos. 

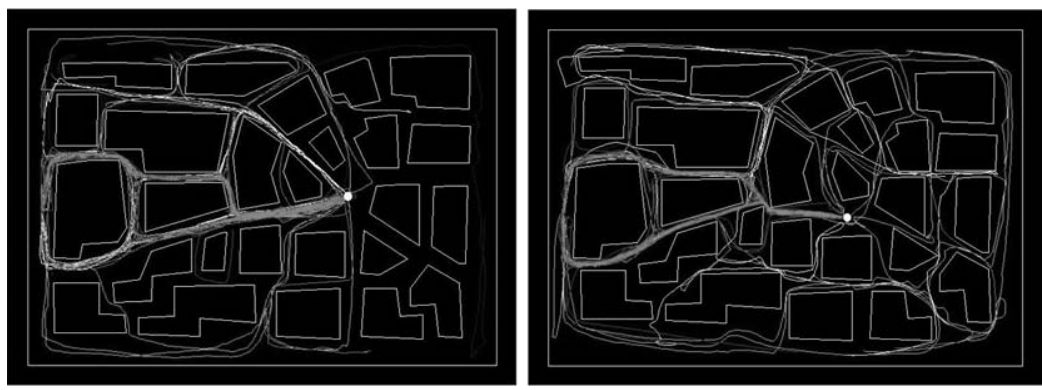

Figura 4: movimiento registrado por las personas en dos mundos virtuales (Fuente: Ruth Conroy-Dalton)

\section{Hacia dónde vamos}

Los puntos anteriores muestran que las teorías contemporáneas de la cognición entienden la relación mente-cuerpo-mundo como un todo integrado, donde es imposible entender los procesos mentales como si estos ocurrieran en un espacio "neutral", ajenos a la acción del cuerpo. Se argumentó también que el medio físico es a la vez causa y consecuencia de estos procesos mentales, pues influencian, y son influenciados por, el comportamiento. El resultado es así un todo dinámico, donde acción y pensamiento se entrelazan en un continuum.

El desafío de la arquitectura está en diseñar ambientes que provean estas claves, para así hacer confluir la triada mente-cuerpo-mundo que permite que nos movamos y orientemos en el medio. Ello implica superar la visión tradicional de la arquitectura de que los edificios y casas deben ser diseñados cuyos propósitos les son del todo claro, y cuyo aparato perceptual es relativamente impermeable a claves ambientales que alteren esos propósitos. En cambio, se piensa que la arquitectura y la planificación urbana debieran hacer suyas la idea de que hay claves perceptuales que afectan el comportamiento y que éste, a su vez, afecta el medio, conformándose un proceso circular de tipo ecológico entre mente y cuerpo. 


\section{Notas}

${ }^{1}$ Los autores agradecen el a Conicyt el apoyo brindado a través del Proyecto Fondecyt Iniciación $\mathrm{N}^{\circ} 11100020$

${ }^{2} \mathrm{Al}$ respecto, el término en inglés “survey knowledge” es también comúnmente utilizado. 


\section{Bibliografía}

Alexander C. (1988), “A city is not a tree” en Thackara, J. (ed.), Design After Modernism: Beyond the Object, Thames and Hudson, London, pp 67-84.

Anderson M. (2003), “Embodied cognition: A field guide”, en Artificial Intelligence, 149, pp 91-130

Batty M. (2005), Cities and Complexity: understanding cities with cellular automata, agent-based models and fractals. MIT Press, Cambridge, Massachussets.

Benedikt M. L. (1979), “To take hold of space: isovist and isovists fields” en Environment and Planning B 6: pp 47-65

Brigthon H., Smith K. \& Kirby S. (2003), "Situated cognition and the role of multi-agent models in explaining language structure” en Kudenko D., Alonso E., Kazakov D. (eds.), Adaptive agents and multi-agent systems: adaptation and multi-agent Learning. Springer, NY.

Burgess N. (2008), "Spatial cognition and the brain" en Annals of New York Academic Science. Mar; 1124:77-97.

Claramunt C. \& Winter S. (2007), "Structural salience of elements of the city” en Environment and Planning B: Planning and Design 34: pp 10301050

Clark A., \& Chalmers D. (1998), “The extended mind” en Analysis, 58: pp 10-23

Conroy-Dalton, R. (2001), Spatial navigation in immersive virtual environments. PhD thesis, Bartlett School of Architecture. University of London, London.

Ídem (2003), “The secret is to follow your nose: route path selection and angularity”. en Environment and Behavior 35(1), pp 107-131

Cornejo C., Ibañez A., Lopez V., (2008), "Significado, contexto y experiencia: Evidencias conductuales y electrofisiológicas del holismo del significado" en C. Cornejo and E. Kronmüller (Ed.), La pregunta por la mente: Aproximaciones desde Latinoamérica. JCSaez Editor, Santiago de Chile.

Cosmelli D, Ibáñez A. (2008), “Human cognition in context: on the biologic, cognitive and social reconsideration of meaning as making sense of action” en Integrated Psychological Behavioral Science. 2008 Jun; 42 (2): pp 233-44. 
Engeström I. (1991), “Activity theory and social transformation” en Multidisiplinary newsletter for activity theory, 7, pp 6-17.

Gärling T., Böök A., et al. (1986), "Spatial orientation and wayfinding in the designed environment: A conceptual analysis and some suggestions for post-occupancy evaluation” en Journal of Architectural and Planning Research 3: pp 55-64

Gibson J. (1979). The ecological approach to visual perception, Houghton Miffin Co.

Golledge R. G., Smith T. R., et al. (1985), “A conceptual model and empirical analysis of children's acquisition of spatial knowledge” en Journal of Environmental Psychology 5:pp 125-152

Golledge R. G. (1992), “Place recognition and wayfinding: making sense of space” en Geoforum 23(2): pp 199-214

Ídem (1995). "Path selection and human preference in human navigation: a progress report” en Conference on Information Theory, COSIT II 1995, Semmering, Austria. Proceedigs. Lecture Notes in Computer Science, [S.l.], v. 988, p. 207-222, 1995.

Golledge, R. G. and R. J. Stimson (1997), Spatial behavior: a geographical perspective. Guilford, New York

Hillier, B. (1996), Space is the machine. Cambridge University Press, Cambridge.

Hillier, B., A. Penn, et al. (1993). "Natural movement: or, configuration and attraction in urban pedestrian movement” en Environment and Planning B: Planning and Design 20: pp 29-66.

Hillier B, (2008), "Space and Spatiality: what the built environment needs from social theory” en. Building Research and Information 36 (3): pp 216-230

Hillier B. \& Vaughan, L. (2007), “The city as one thing” en Progress in Planning, 67 (3): pp 205 - 230

Hölscher C., Buchner S., Meilinger T., Strube G. (2007), “Map use and wayfinding strategies in a multi-building ensemble” en Spatial Cognition V: Reasoning, Action, Interaction, Bremen, Germany, Lecture Notes in Artificial Intelligence. 4387: pp 365-380

Harvey D. (1973), Social Justice and the City, Edward Arnold and The John Hopkins University Press.

Hutchins E. (1995), Cognition in the Wild. The MIT Press, Boston. 
Ibáñez A, Cosmelli D. (2008), “Moving beyond computational cognitivism: understanding intentionality, intersubjectivity and ecology of mind” en Integrated Psychological Behavioral Science 42(2): pp 129-36.

Ibáñez. A. (2008), Dynamics of Cognition, JCSaez, Santiago de Chile.

Ídem (2007a), “The Dynamic Core of Consciousness and Neural Darwinism” en Rev Neurol (Behavioral Neurology), 45 (9), pp 547-555

Ídem (2007b), “Complexity and Cognition: Mind and Brain as a Topological Dynamical System” en Nonlinear Dynamics, Psychology, and Life Sciences. 11(1): pp 51-90.

Ibáñez A., Haye A., González R., Hurtado E., Henríquez, R. (2009), “Multilevel analysis of cultural phenomena: The role of ERP approach to prejudice" en The Journal for Theory in Social Behavior, 39, pp 81-110.

Ibáñez A., Lopez V., Cornejo C. (2006), “ERPs and contextual semantic discrimination: Evidence of degrees of congruency in wakefulness and sleep” en Brain and Language, 98, (3), pp 264-275.

Kaplan, S. \& R. Kaplan (1982), Cognition and environment: functioning in an uncertain world. Ulrich's, Michigan.

Lakoff G., \& Johnson M. (1999), Philosophy in the Flesh: The embodied mind and its challenge to western thought. Basic Books, New York

Lynch K. (1960), The image of the city. Boston, MIT Press

Marshall S. (2004), Street and patterns, Spon Press.

Mora R, (2010), “Moviéndose con la mente y el cuerpo: entendiendo la diferencia entre planear y realizar rutas en el medio construido" en Revista Fractal 22 (2): pp 373-384.

Muir GM, Taube JS. (2002), “The neural correlates of navigation: do head direction and place cells guide spatial behavior?” en Behavioral Cognitive Neuroscience Review. 1(4): pp 297-317

Nakazaw, K, Thomas J., Matthew A. Wilson \& Susumu Tonegawa. (2004), "NMDA receptors, place cells and hippocampal spatial memory" en Nature Reviews Neuroscience 5: pp 361-372.

Portugali J. (2004), “Toward a cognitive approach to urban dynamics” en Environment and Planning B: Planning and Design 31: pp 589-613

Siegel A. W. \&. White S. H .(1975), "The development of spatial representations of large-scale environments” en Advances in Child 
Development and Behavior. H. Reese. New York, Academic Press. 10: 955

Soja E. (1989). Postmodern Geographies: The Reassertion of Space in Critical Social Theory. London: Verso Press

Salingaros N. (2005), "Principios de estructura urbana” en Design Science Planning, 2005. The Theory, Science and Practice of Bringing Buildings to Life, Kellert, S R., Heerwagen, J. y Mador, M (editores). John Wiley, New York, pp 59-83

Thompson E., \& Varela F. (2001), "Radical embodiment: Neural dynamics and consciouness” en Trends in Cognitive Sciences 10, pp 418-425.

Tolman E. C. (1948), “Cognitive maps in rats and men” en Psychological review 55(4): pp 189-208.

Recibido: 25.11.2010

Aceptado: 20.07.2011 